\title{
GAP ESTIMATES OF SCHRÖDINGER OPERATOR
}

\author{
Roger Chen
}

\begin{abstract}
By a transformation of metric using the first eigenfunction, we obtain lower bounds for all eigenvalues of Schrödinger operator with the Neumann boundary condition. Global estimates for first eigenfunction are needed and this is achieved by the Moser iteration technique.
\end{abstract}

\section{Introduction.}

Let $\left(M^{m}, d s^{2}\right)$ be an $m$-dimensional compact Riemannian manifold with metric $d s^{2}$ and $\partial M \neq \emptyset$ be the boundary of $M$. Let $\Delta$ be the Laplacian operator associated to $d s^{2}$ on $M$ then we define on $M$ a Schrödinger operator by

$$
\Delta-q(x)
$$

where $q(x) \in C^{2}(M)$. In this paper, we consider the following Neumann eigenvalue problem:

$$
\begin{aligned}
\Delta u-q u & =-\eta u & & \text { in } M \\
\frac{\partial u}{\partial \nu} & =0 & & \text { on } \partial M .
\end{aligned}
$$

It is well known that the sets of eigenvalues $\left\{\eta_{k}\right\}$ can be arranged in a nondecreasing order of magnitude as follows:

$$
\eta_{1} \leq \eta_{2} \leq \cdots \leq \eta_{k} \leq \cdots \rightarrow \infty
$$

When $q \equiv 0$ in (1), the lower estimate of $\eta_{1}$ corresponds to the important Poincaré inequality. Analogously, for nontrivial $q$, it is interesting to find a lower bound for the gap of $\eta_{2}-\eta_{1}$, or $\eta_{k}-\eta_{1}$ in terms of the geometrical quantities of $M$ and the potential function $q$. Since the boundary $\partial M$, in general, is not a zero level set of the first eigenfunction, one needs some convexity assumptions on $\partial M$ in order to obtain a positive lower estimate for the first Neumann eigenvalue (cf. Cheeger's example in $[\mathbf{C}]$ ). These eigenvalue problems have been studied by a long list of authors. We will simply refer the reader to Buser $[\mathbf{B}]$, Cheeger $[\mathbf{C}]$, Chen [Cn1], Chen-Li [CnL], Cheng-Li [CL], Payne-Weinberger [PW], Li [L1], [L2], Li-Yau [LY1], [LY2], Meyer 
[M], Singer-Wong-Yau-Yau [SWYY], Wang [W], and Zhong-Yang [ZY] for further references. The purpose of this paper is to show that one can apply a similar argument as used by Cheng-Li $[\mathbf{C L}]$ to prove following generalization of a Wang's result $[\mathbf{W}]$.

Theorem 1. Let $M^{m}, m \geq 3$, be an m-dimensional compact manifold with boundary $\partial M$ and $\omega(q)=\sup q-\inf q$ denote the oscillation of $q$ over $M$. Suppose that the Ricci curvature of $M$ satisfies $\operatorname{Ric}_{M} \geq-K, K \geq 0$ and the second fundamental form elements of $\partial M$ with respect to outward pointing unit normal $\nu$ satisfies $I I \geq-H, H \geq 0$. Suppose that $\partial M$ also satisfies the "interior rolling $R$-ball condition" with $R$ chosen small (see $[\mathbf{C n} 1],[\mathbf{W}]$ for the choice of $R$ ). Then the gap $\Gamma_{k}$ of $k$-th Neumann eigenvalues $\eta_{k}$ and $\eta_{1}$ of $M$ satisfies

$$
\Gamma_{k} \geq \alpha_{1} k^{\frac{2}{m}}
$$

for all $k=2,3, \ldots$ and some constant $\alpha_{1}$ depending on $m, K, H, R, d$ and $\omega(q)$.

Remark. Note that only the $C^{0}$ norm, instead of the $C^{2}$ norm, of the potential function $q$ is needed to obtain the estimates. It is different from that when one employs gradient estimates and maximum principle type argument (cf. [Cn2]).

Acknowledgments. We would like to thank Professors Alice Chang, Peter Li, Jiaping Wang and the referee for their helpful suggestions.

\section{Preliminaries.}

Let $\left(M^{m}, g\right), m \geq 3$, be an $m$-dimensional compact Riemannian manifold with boundary $\partial M$. Let $\frac{\partial}{\partial \nu}$ be the outward pointing unit normal vector to $\partial M$, and denote the second fundamental form of $\partial M$ with respect to $\frac{\partial}{\partial \nu}$ by II. We recall the following definition from [Cn1].

Definition 1. $\partial M$ is said to satisfy the "interior rolling $R$-ball" condition if for each point $p \in \partial M$ there is a geodesic ball $B_{q}\left(\frac{R}{2}\right)$, centered at $q \in M$ with radius $\frac{R}{2}$, such that $p=B_{q}\left(\frac{R}{2}\right) \cap \partial M$ and $B_{q}\left(\frac{R}{2}\right) \subset M$.

It is proved independently in $[\mathbf{C n} \mathbf{1}]$ and $[\mathbf{M}]$, (also see $[\mathbf{W}]$ ), that

Theorem 2. Let $M$ be as in Theorem 1. Then the first nonzero Neumann eigenvalue $\lambda_{1}$ of Laplacian operator has a lower bound given by

$$
\lambda_{1} \geq C_{p}=\frac{1}{(1+H)^{2}}\left(\frac{1-\alpha^{2}}{4(m-1) d^{2}} B_{1}^{2}-B_{2}\right) \exp \left(-B_{1}\right),
$$


$\alpha$ and $R$ are positive constants less than 1 ,

$$
\begin{aligned}
& d=\text { diameter of } M, \\
& B_{1}=1+\left[1+\frac{4(m-1) d^{2} C}{1-\alpha^{2}}\right]^{\frac{1}{2}}, \\
& B_{2}=(1+H) B_{3}+\frac{\left[(2 m-3)^{2}+(4 m-5) \alpha^{2}\right] H^{2}}{(m-1) R^{2} \alpha^{2}}+(1+H)^{2} K, \\
& B_{3}=\frac{2(m-1) H(1+H)(1+3 H)}{R}+\frac{H(1+H)}{R^{2}} .
\end{aligned}
$$

For all $f \in C^{\infty}(\bar{M})$ satisfying $\int_{M} f=0$, Wang [W] proves that the following Neumann type of Sobolev inequality

$$
\left(\int_{M}|f|^{\frac{2 m}{m-2}}\right)^{\frac{m-2}{m}} \leq C(S) \int_{M}|\nabla f|^{2}
$$

holds on the manifold $M$ as in Theorem 1 and the minimum constant $C(S)$, called the Neumann Sobolev constant of $M$, can be estimated as follows.

Theorem 3. Let $M$ be as in Theorem 1. Then the Neumann Sobolev constant $C(S)$ of $M$ satisfies

$$
C(S) \leq C(m) A^{\frac{2}{m}}
$$

where $C(m)$ is a constant only depending on $m$ and $A=A(d, H, R, K, m)$ is a positive constant which can be explicitly computed.

Corollary 1. Let $M$ be as in Theorem 1 and $\alpha_{2}=C(m) A^{\frac{2}{m}}$. For all $f \in C^{\infty}(\bar{M})$,

$$
\int_{M}|\nabla f|^{2} \geq \frac{1}{\alpha_{2}}\left[\frac{1}{2}\left(\int_{M}|f|^{\frac{2 m}{m-2}}\right)^{\frac{m-2}{m}}-V(M)^{-\frac{2}{m}} \int_{M}|f|^{2}\right]
$$

holds on $M$.

Proof. Let $f \in C^{\infty}(\bar{M})$ and $a=\frac{1}{V(M)} \int_{M} f$. Using Minkowski's inequality and Hölder's inequality, we get that

$$
\begin{aligned}
\left(\int_{M}|f|^{\frac{2 m}{m-2}}\right)^{\frac{m-2}{m}} & \leq 2\left(\int_{M}|f-a|^{\frac{2 m}{m-2}}\right)^{\frac{m-2}{m}}+2\left(\int_{M}|a|^{\frac{2 m}{m-2}}\right)^{\frac{m-2}{m}} \\
& \leq 2\left(\int_{M}|f-a|^{\frac{2 m}{m-2}}\right)^{\frac{m-2}{m}}+2 V(M)^{-\frac{2}{m}} \int_{M}|f|^{2}
\end{aligned}
$$

We apply the Sobolev inequality (2) to the function $f-a$ to get 


$$
\begin{aligned}
\int_{M}|\nabla f|^{2} & \geq \frac{1}{\alpha_{2}}\left(\int_{M}|f-a|^{\frac{2 m}{m-2}}\right)^{\frac{m-2}{m}} \\
& \geq \frac{1}{\alpha_{2}}\left[\frac{1}{2}\left(\int_{M}|f|^{\frac{2 m}{m-2}}\right)^{\frac{m-2}{m}}-V(M)^{-\frac{2}{m}} \int_{M}|f|^{2}\right] .
\end{aligned}
$$

This proves the corollary.

Applying the above Sobolev inequality (3) and the estimate on the first Neumann eigenvalue of Laplacian in the Moser iteration scheme, we get a global Harnack inequality for the first Neumann eigenfunction of Schrödinger operator.

Theorem 4. Let $M$ be as in Theorem 1. Assume that $u(x)$ is a first Neumann eigenfunction on $M$ satisfying

$$
\begin{aligned}
(\Delta-q) u & =-\eta_{1} u \\
\left.\frac{\partial u}{\partial \nu}\right|_{\partial M} & =0
\end{aligned}
$$

then for any $x, y \in M$, we have

$$
u_{1}(x) \leq C_{h} u_{1}(y),
$$

where $C_{h}(M)>1$ is a constant depending on $m, V(M), C_{p}, \alpha_{2}, \omega(q)$, and it can be explicitly computed.

Proof. By modifying arguments in $[\mathbf{L} 4]$, it suffices to prove the following two lemmas.

Lemma 1. Let $M, u$ be as in the theorem. For any $k>0$, there exists a positive constant

$$
\alpha_{3}=\left[\left(2 k \omega(q) \alpha_{2}\right)^{\frac{m}{2 k}}+2^{\frac{m}{k}} V(M)^{\frac{-1}{k}}\right]\left(\frac{m}{m-2}\right)^{\frac{1}{k} \sum_{j=0}^{\infty} j\left(\frac{m-2}{m}\right)^{j}}
$$

such that

$$
\|u\|_{\infty} \leq \alpha_{4} \# u \|_{k}
$$

where $\alpha_{4}=\max \left\{\alpha_{3},\left(\alpha_{3}^{2} V(M)\right)^{\frac{1}{k}}\right\},\|u\|_{\infty}$ denotes the $L^{\infty}$ norm of $u,\|u\|_{k}=$ $\left(\int_{M}|u|^{k}\right)^{\frac{1}{k}}$ and $\# u \|_{k}=\left(\frac{1}{V(M)} \int_{M}|u|^{k}\right)^{\frac{1}{k}}$.

Proof. For any constant $a \geq 1$ and $\mu=\frac{m}{m-2}$ the assumption of $u$ implies that

$$
\omega(q) \int_{M} u^{2 a} \geq-\int_{M} u^{2 a-1} \Delta u
$$


Integrating by parts and using the boundary condition, the right hand side yields

$$
\begin{aligned}
-\int_{M} u^{2 a-1} \Delta u & =(2 a-1) \int_{M} u^{2 a-2}|\nabla u|^{2} \\
& \geq a \int_{M} u^{2 a-2}|\nabla u|^{2} .
\end{aligned}
$$

The Sobolev inequality (3) implies that

$$
\begin{aligned}
& a^{2} \int_{M} u^{2 a-2}|\nabla u|^{2} \\
& =\int_{M}\left|\nabla u^{a}\right|^{2} \\
& \geq \frac{1}{\alpha_{2}}\left[\frac{1}{2}\left(\int_{M} u^{2 a \mu}\right)^{\frac{1}{\mu}}-V(M)^{-\frac{2}{m}} \int_{M} u^{2 a}\right] .
\end{aligned}
$$

By setting $\alpha_{5}=2 \alpha_{2} \omega(q) a+2 V(M)^{-\frac{2}{m}}$, we get

$$
\left(\int_{M} u^{2 a \mu}\right)^{\frac{1}{\mu}} \leq \alpha_{5} \int_{M} u^{2 a}
$$

which is equivalent to

$$
\alpha_{5}^{\frac{1}{2 a}}\|u\|_{2 a} \geq\|u\|_{2 a \mu}
$$

Let us choose a sequence of $a_{i}$ such that

$$
a_{0}=\frac{k}{2}, a_{1}=\frac{k \mu}{2}, \cdots, a_{i}=\frac{k \mu^{i}}{2}, \cdots
$$

Applying (6) to $a=a_{i}$ and iterating the inequality, we conclude that

$$
\|u\|_{2 a_{i+1}} \leq \prod_{j=0}^{i}\left(2 a_{j} \omega(q) \alpha_{2}+2 V(M)^{-\frac{2}{m}}\right)^{\frac{1}{2 a_{j}}}\|u\|_{k} .
$$

On the other hand, we have

$$
\lim _{i \rightarrow \infty} \# u\left\|_{2 a_{i+1}}=\right\| u \|_{\infty}
$$

Therefore, letting $i \rightarrow \infty$, we conclude that

$$
\|u\|_{\infty} \leq \prod_{j=0}^{\infty}\left(k \mu^{j} \omega(q) \alpha_{2}+2 V(M)^{-\frac{2}{m}}\right)^{\frac{1}{k \mu^{j}}}\|u\|_{k} .
$$


The product can be estimated by using the fact that

$$
\prod_{j=0}^{\infty} B^{\mu^{-j}}=B^{\frac{\mu}{\mu-1}}
$$

and the fact that $\sum_{j=0}^{\infty} j \mu^{-j}$ is finite. Hence we have

$$
\begin{aligned}
& \prod_{j=0}^{\infty}\left(k \mu^{j} \omega(q) \alpha_{2}+2 V(M)^{-\frac{2}{m}}\right)^{\frac{1}{k \mu j}} \\
& \leq \prod_{j=0}^{\infty}\left[\left(k \omega(q) \alpha_{2}+2 V(M)^{-\frac{2}{m}}\right) \mu^{j}\right]^{\frac{1}{k \mu^{j}}} \\
& \leq\left(k \omega(q) \alpha_{2}+2 V(M)^{-\frac{2}{m}}\right)^{\frac{\mu}{k(\mu-1)}} \mu^{\frac{1}{k} \sum_{j=0}^{\infty} j \mu^{-j}} \\
& \leq\left[\left(2 k \omega(q) \alpha_{2}\right)^{\frac{m}{2 k}}+2^{\frac{m}{k}} V(M)^{\frac{-1}{k}}\right]\left(\frac{m}{m-2}\right)^{\frac{1}{k} \sum_{j=0}^{\infty} j\left(\frac{m-2}{m}\right)^{j}} \\
& =\alpha_{3} .
\end{aligned}
$$

We have proved that

$$
\|u\|_{\infty} \leq \alpha_{3} V(M)^{\frac{1}{k}}+u \|_{k}
$$

for $a \geq 1$, or equivalently, for $k \geq 2$. For those values $k<2$, we begin with the case $k=2$. In that case, the inequality takes the form

$$
\begin{aligned}
\|u\|_{\infty} & \leq \alpha_{3} V(M)^{\frac{1}{2}} \# u \|_{2} \\
& \leq \alpha_{3}\|u\|_{k}^{\frac{k}{2}}\|u\|_{\infty}^{1-\frac{k}{2}} .
\end{aligned}
$$

Iterating the inequality yields

$$
\|u\|_{\infty} \leq\|u\|_{\infty}^{\left(1-\frac{k}{2}\right)^{i}} \prod_{j=1}^{i}\left[\alpha_{3}\|u\|_{k}^{\frac{k}{2}}\right]^{\left(1-\frac{k}{2}\right)^{j-1}} .
$$

Letting $i \rightarrow \infty$, the term

$$
\|u\|_{\infty}^{\left(1-\frac{k}{2}\right)^{i}} \rightarrow 1
$$

and

$$
\prod_{j=1}^{\infty}\|u\|_{k}^{\frac{k}{2}\left(1-\frac{k}{2}\right)^{j-1}}=\|u\|_{k}
$$


Hence, (8) implies that

$$
\|u\|_{\infty} \leq\left(\alpha_{3}^{2} V(M)\right)^{\frac{1}{k}} \# u \|_{k}
$$

This proves our lemma.

Lemma 2. Let $M, u$ be as in the theorem. For $k>0$ sufficiently small, there exists constant $\alpha_{10}$ depending on $m, k, V(M), C_{p}, \alpha_{2}$ and $\omega(q)$ such that

$$
\begin{aligned}
\# u \|_{k} & \leq \frac{1}{\alpha_{4}^{2} \alpha_{10}^{2}} \inf _{M} u \\
& =C_{h} \inf _{M} u .
\end{aligned}
$$

Proof. The function $u^{-1}$ satisfies that

$$
\begin{aligned}
\Delta u^{-1} & =-u^{-2} \Delta u+2 u^{-3}|\nabla u|^{2} \\
& \geq-\omega(q) u^{-1}
\end{aligned}
$$

By applying Lemma 1 to $u^{-1}$, we have

$$
\begin{aligned}
\left(\inf _{M} u\right)^{-1} & =\sup _{M} u^{-1} \\
& \leq \alpha_{4} \# u^{-1} \|_{k} .
\end{aligned}
$$

Clearly, the lemma follows if we can estimate the product

$$
\# u^{-1}\left\|_{k} \cdot \# u\right\|_{k}
$$

from above for some value of $k>0$. To achieve this, let us consider the function

$$
v=\beta+\log u
$$

where $\beta=\frac{-1}{V(M)} \int_{M} \log u$. The function $v$ satisfies

$$
\begin{aligned}
\Delta v & =\frac{\Delta u}{u}-\frac{|\nabla u|^{2}}{u^{2}} \\
& \leq \omega(q)-|\nabla v|^{2}
\end{aligned}
$$

hence

$$
|\nabla v|^{2} \leq \omega(q)-\Delta v
$$


Integrating and using the boundary condition of $u$, we deduce that

$$
\int_{M}|\nabla v|^{2} \leq \omega(q) V(M) .
$$

However, the estimate of the first Neumann eigenvalue and the choice of $\beta$ implies that

$$
C_{p} \int_{M} v^{2} \leq \int_{M}|\nabla v|^{2}
$$

Hence, we have

$$
\int_{M} v^{2} \leq \frac{\omega(q) V(M)}{C_{p}}
$$

Applying the Schwarz inequality, we also have

$$
\int_{M}|v| \leq\left(\frac{\omega(q)}{C_{p}}\right)^{\frac{1}{2}} V(M) .
$$

Multiplying $|v|^{2 a-2}$ to (11) for $a \geq 2$, and integrating by parts yields

$$
\begin{aligned}
& \int_{M}|v|^{2 a-2}|\nabla v|^{2} \\
& \leq \omega(q) \int_{M}|v|^{2 a-2}-\int_{M}|v|^{2 a-2} \Delta v \\
& \leq \omega(q) \int_{M}|v|^{2 a-2}+(2 a-2) \int_{M}|v|^{2 a-3}|\nabla v|^{2} .
\end{aligned}
$$

Using (12) and the inequality

$$
(2 a-2) \int_{M}|v|^{2 a-3}|\nabla v|^{2} \leq \frac{1}{4} \int_{M}|v|^{2 a-2}|\nabla v|^{2}+(8 a-12)^{2 a-3} \int_{M}|\nabla v|^{2},
$$

(15) becomes

$$
\begin{aligned}
& \int_{M}|v|^{2 a-2}|\nabla v|^{2} \\
& \leq 2 \omega(q) \int_{M}|v|^{2 a-2}+2(8 a-12)^{2 a-3} \int_{M}|\nabla v|^{2} \\
& \leq 2 \omega(q)\left(\int_{M}|v|^{2 a-2}+V(M)(8 a-12)^{2 a-3}\right) .
\end{aligned}
$$

By setting $a=2$ and combining with (13), we have

$$
\int_{M} v^{2}|\nabla v|^{2} \leq \alpha_{6}
$$


for some constant $\alpha_{6}=\frac{2 \omega(q)^{2} V(M)}{C_{p}}+8 \omega(q) V(M)$. On the other hand,

$$
\begin{aligned}
\int_{M} v^{2}|\nabla v|^{2} & =\frac{1}{4} \int_{M}\left|\nabla \operatorname{sgn}(v) v^{2}\right|^{2} \\
& \geq \frac{\lambda_{1}(M)}{4} \int_{M}\left(\operatorname{sgn}(v) v^{2}-\frac{1}{V(M)} \int_{M} \operatorname{sgn}(v) v^{2}\right)^{2} \\
& \geq \frac{C_{p}}{4}\left\{\int_{M}|v|^{4}-\frac{1}{V(M)}\left(\int_{M} \operatorname{sgn}(v) v^{2}\right)^{2}\right\} \\
& \geq \frac{C_{p}}{4}\left\{\int_{M}|v|^{4}-\frac{1}{V(M)}\left(\int_{M} v^{2}\right)^{2}\right\}
\end{aligned}
$$

Hence combining with (16), we have

$$
\int_{M}|v|^{4} \leq \alpha_{7}
$$

for some constant $\alpha_{7}=\frac{9 \omega(q)^{2} V(M)}{C_{p}^{2}}+\frac{32 \omega(q) V(M)}{C_{p}}$. Using Schwarz inequality, we also deduce that

$$
\int_{M}|v|^{3} \leq \alpha_{7}^{\frac{3}{4}} V(M)^{\frac{1}{4}} .
$$

For general $a \geq 2$, note that

$$
\left|\nabla\left(|v|^{a}\right)\right|^{2}=a^{2}|v|^{2 a-2}|\nabla v|^{2}
$$

Combining this with (16), we conclude that

$$
\int_{M}\left|\nabla\left(|v|^{a}\right)\right|^{2} \leq 2 a^{2} \omega(q)\left(\int_{M}|v|^{2 a-2}+V(M)(8 a-12)^{2 a-3}\right) .
$$

Using the inequality

$$
|v|^{2 a-2} \leq|v|^{2 a}+1
$$

we have

$$
\int_{M}\left|\nabla\left(|v|^{a}\right)\right|^{2} \leq \omega(q)\left(2 a^{2} \int_{M}|v|^{2 a}+V(M)(8 a-12)^{2 a-1}\right) .
$$

Hence, applying Sobolev inequality (3),

$$
\frac{1}{\alpha_{2}}\left[\frac{1}{2}\left(\int_{M}\left(|v|^{a}\right)^{2 \mu}\right)^{\frac{1}{\mu}}-V(M)^{-\frac{2}{m}} \int_{M}\left(|v|^{a}\right)^{2}\right] \leq \int_{M}\left|\nabla\left(|v|^{a}\right)\right|^{2},
$$


we conclude that

$$
\begin{aligned}
\left(\int_{M}|v|^{2 a \mu}\right)^{\frac{1}{2 a \mu}} \leq & \left(4 a^{2} \omega(q) \alpha_{2}+2 V(M)^{-\frac{2}{m}}\right)^{\frac{1}{2 a}}\left(\int_{M}|v|^{2 a}\right)^{\frac{1}{2 a}} \\
& +\left(2 \omega(q) V(M) \alpha_{2}\right)^{\frac{1}{2 a}}(8 a) .
\end{aligned}
$$

Consider the sequence

$$
a_{0}=2, a_{1}=2 \mu, \cdots, a_{i}=2 \mu^{i}, \cdots .
$$

Applying the inequality to $a_{i}$, we have

$$
\begin{aligned}
\left(\int_{M}|v|^{4 \mu^{i+1}}\right)^{\frac{1}{4 \mu^{i+1}}} \leq & \left(4 \omega(q) \alpha_{2}+2 V^{-\frac{2}{m}}\right)^{\frac{1}{4 \mu^{i}}}\left(2 \mu^{i}\right)^{\frac{1}{2 \mu^{i}}}\left(\int_{M}|v|^{4 \mu^{i}}\right)^{\frac{1}{4 \mu^{i}}} \\
& +\left(2 \omega(q) V(M) \alpha_{2}\right)^{\frac{1}{4 \mu^{i}}}\left(16 \mu^{i}\right) .
\end{aligned}
$$

Iterating this by running $i=0, \cdots, l$ gives

$$
\begin{aligned}
& \|v\|_{4 \mu^{l+1}} \\
\leq & \prod_{i=0}^{l}\left(4 \omega(q) \alpha_{2}+2 V^{-\frac{2}{m}}\right)^{\frac{1}{4 \mu^{i}}}\left(2 \mu^{i}\right)^{\frac{1}{2 \mu^{i}}}\|v\|_{4}+\left(2 \omega(q) V(M) \alpha_{2}\right)^{\frac{1}{4 \mu^{l}}}\left(16 \mu^{l}\right) \\
+ & \sum_{i=0}^{l-1}\left(2 \omega(q) V(M) \alpha_{2}\right)^{\frac{1}{4 \mu^{i}}}\left(16 \mu^{i}\right) \prod_{j=i+1}^{l}\left(4 \omega(q) \alpha_{2}+2 V^{-\frac{2}{m}}\right)^{\frac{1}{4 \mu^{j}}}\left(2 \mu^{j}\right)^{\frac{1}{2 \mu^{j}}}
\end{aligned}
$$

Using the equality $\sum_{i=0}^{\infty} \mu^{-i}=\frac{m}{2}$, and the fact that $\sum_{i=0}^{\infty} i \mu^{-i}$ is finite, we conclude that

$$
\begin{aligned}
& \mathbb{H} \|_{4 \mu^{l+1}} \\
& \leq\left\{16\left(8 \omega(q) \alpha_{2}\right)^{\frac{m}{8}}+2^{\frac{m}{4}} V^{\frac{-1}{4}}\right\}\left(\frac{m}{m-2}\right)^{\frac{1}{k} \sum_{0}^{\infty} i\left(\frac{m-2}{m}\right)^{i}}\left(\# v \|_{4}+\sum_{i=0}^{l} \mu^{i}\right) \\
& \leq \alpha_{8}\left(\# v \|_{4}+4 \mu^{l}\right) .
\end{aligned}
$$

For each integer $j \geq 4$, let $l$ be such that $4 \mu^{l}<j<4 \mu^{l+1}$. Using the Hölder inequality and the estimate (19), we get

$$
\begin{aligned}
f_{M}|v|^{j} & \leq\left(f_{M}|v|^{4 \mu^{l+1}}\right)^{\frac{j}{4 \mu^{l+1}}} \\
& \leq \alpha_{8}^{j}\left(\# v \|_{4}+j\right)^{j} .
\end{aligned}
$$


Combining this with (13), (14), (17) and (18), we have

$$
\begin{aligned}
f_{M} e^{k|v|} & =\sum_{j=0}^{\infty} \frac{k^{j}}{j !} f_{M}|v|^{j} \\
& \leq\left(1+\sum_{j=1}^{4} \frac{k^{j}}{j !} \alpha_{7}^{\frac{j}{4}} V(M)^{\frac{j}{4}-1}\right)+\sum_{j=5}^{\infty} \frac{\left[\alpha_{8}\left(\alpha_{7}^{\frac{1}{4}} V(M)^{\frac{-1}{4}}+1\right) k j\right]^{j}}{j !} \\
& \leq \alpha_{9}+\sum_{j=5}^{\infty} \frac{\left[\alpha_{8}\left(\alpha_{7}^{\frac{1}{4}} V(M)^{\frac{-1}{4}}+1\right) k j\right]^{j}}{j !} .
\end{aligned}
$$

However, using Stirling's inequality $j^{j}<j$ ! $e^{j}$, we conclude that

$$
f_{M} e^{k|v|} \leq \alpha_{9}+\sum_{j=5}^{\infty}\left[\alpha_{8}\left(\alpha_{7}^{\frac{1}{4}} V(M)^{\frac{-1}{4}}+1\right) k e\right]^{j}
$$

Therefore, by choosing $k<\left[\alpha_{8}\left(\alpha_{7}^{\frac{1}{4}} V(M)^{\frac{-1}{4}}+1\right) e\right]^{-1}$, the infinite series converges and we obtain the estimate

$$
f_{M} e^{k|v|} \leq \alpha_{10}
$$

where $\alpha_{10}=\alpha_{9}+\sum_{j=5}^{\infty}\left[\alpha_{8}\left(\alpha_{7}^{\frac{1}{4}} V(M)^{\frac{-1}{4}}+1\right) k e\right]^{j}$. Let us now observe that

$$
\begin{aligned}
e^{k \beta} u^{k} & =e^{k v} \\
& \leq e^{k|v|}
\end{aligned}
$$

and

$$
\begin{aligned}
e^{-k \beta} u^{-k} & =e^{-k v} \\
& \leq e^{k|v|}
\end{aligned}
$$

imply that

$$
\# u^{-1}\left\|_{k} \cdot H u\right\|_{k} \leq\left(f_{M} e^{k|v|}\right)^{2}
$$


This implies that

$$
\begin{aligned}
\inf _{M} u & \geq \frac{1}{\alpha_{4} \# u^{-1} \|_{k}} \\
& \geq \frac{1}{\alpha_{4} \alpha_{10}^{2}} \# u \|_{k} \\
& \geq \frac{1}{\alpha_{4}^{2} \alpha_{10}^{2}} \sup _{M} u .
\end{aligned}
$$

\section{Proof of Theorem 1.}

In this section, we shall utilize Harnack inequality to prove our Theorem 1. Let $u_{k}$, be the k-th eigenfunction with eigenvalues $\eta_{k}$, for the Equation (1). By setting $\Gamma_{k}=\eta_{k}-\eta_{1}, r_{k}=\frac{u_{k}}{u_{1}}$ and $h=\log u_{1}$, we get that

$$
\begin{aligned}
\Delta r_{k}+2\left\langle\nabla h, \nabla r_{k}\right\rangle & =-\Gamma_{k} r_{k} & & \text { in } M \\
\frac{\partial r_{k}}{\partial \nu} & =0 & & \text { on } \partial M .
\end{aligned}
$$

We consider a new metric $d s_{1}^{2}$ defined by

$$
d s_{1}^{2}=u_{1}^{\frac{4}{m-2}} d s^{2}
$$

with the volume element $d v_{1}=u_{1}^{\frac{2 m}{m-2}} d v$. We shall need the following version of the Sobolev and Poincaré inequalities on $M$.

Lemma 3. Let $M$ be as in Theorem 1. For all $f \in C^{\infty}(\bar{M})$ satisfying that $\int_{M} f d v=0$,

$$
\left(\int_{M}|f|^{\frac{2 m}{m-2}} u_{1}^{-\frac{4}{m-2}} d v_{1}\right)^{\frac{m-2}{m}} \leq \alpha_{11} \int_{M}\left|\nabla_{1} f\right|^{2} d v_{1}
$$

and

$$
\int_{M}|f|^{2} u_{1}^{-\frac{4}{m-2}} d v_{1} \leq \alpha_{12} \int_{M}\left|\nabla_{1} f\right|^{2} d v_{1}
$$

hold on the manifold $M$ for $\alpha_{11}=\alpha_{2}\left(C_{h}^{2} V(M)\right)^{\frac{2 m-2}{m}}$ and $\alpha_{12}=\frac{C_{h}^{4} V(M)^{2}}{C_{p}}$.

Proof. Using $\int_{M} u_{1}^{2} d v=1$ and the Harnack inequality, we can get

$$
\frac{1}{C_{h} \sqrt{V(M)}} \leq u_{1} \leq C_{h} \sqrt{V(M)} .
$$


Combining these with the Theorem 2 and Lemma 1, we prove our lemma.

Now, we shall write the Equation (1) in terms of metric $d s_{1}^{2}$ to get

$$
\begin{aligned}
u_{1}^{\frac{4}{m-2}} \Delta_{1} r & =-\Gamma r & & \text { in } M \\
\frac{\partial}{\partial \nu} r & =0 & & \text { on } \partial M,
\end{aligned}
$$

where $\Delta_{1}$ denotes the Laplacian operator with respect to the metric $d s_{1}^{2}$. Let $W(x, y, t)$ denote the fundamental solution of the equation

$$
\begin{aligned}
\left(u_{1}^{\frac{4}{m-2}} \Delta_{1}-\frac{\partial}{\partial t}\right) w(x, t) & =0 & & \text { in } M \\
\frac{\partial}{\partial \nu} w(x, t) & =0 & & \text { on } \partial M .
\end{aligned}
$$

Following S.Y. Cheng and P. Li [CL], we shall need the following lemma.

Lemma 4. Let $M$ be as in Theorem 1. There exists a constant $\alpha_{13}$ depending on the $m, V(M), C_{h}, C_{p}$ and $\alpha_{2}$ such that

$$
\int_{M}\left|\nabla_{1} f\right|^{2} d v_{1} \geq \alpha_{13}\left(\int_{M} f^{2} d v_{1}\right)^{\frac{m+2}{m}}\left(\int_{M}|f| u_{1}^{\frac{-4}{m-2}} d v_{1}\right)^{\frac{-4}{m}}
$$

for any $f \in C^{\infty}(M)$ which satisfies

$$
\int_{M} f u_{1}^{\frac{-4}{m-2}} d v_{1}=0
$$

Proof. For each $f \in C^{\infty}(M)$, Sobolev inequality (3) implies that

$$
\begin{aligned}
\int_{M}\left|\nabla_{1} f\right|^{2} d v_{1} \geq & \left(C_{h}^{2} V(M)\right)^{-2} \frac{1}{2 \alpha_{2}}\left(\int_{M}|f|^{\frac{2 m}{m-2}} d v_{1}\right)^{\frac{m-2}{m}} \\
& -V(M)^{\frac{-2}{m}} \frac{1}{\alpha_{2}} \int_{M} f^{2} u_{1}^{-\frac{4}{m-2}} d v_{1}
\end{aligned}
$$

Applying Lemma 3, we get

$$
\int_{M}\left|\nabla_{1} f\right|^{2} d v_{1} \geq \alpha_{14}\left(\int_{M}|f|^{\frac{2 m}{m-2}} d v_{1}\right)^{\frac{m-2}{m}}
$$

for some constant $\alpha_{14}>0$ depends on $C_{h}, V(M)$, and $\alpha_{2}$. The lemma is proved by using Hölder's inequality and Theorem 4 . 
Proof of Theorem 1. We consider the function $G(x, y, t)$ defined on $M \times$ $M \times[0, \infty)$ by

$$
G(x, y, t)=W(x, y, t)-1 .
$$

Since $W(x, y, t)$ has the eigenfunction expansion

$$
W(x, y, t)=\sum_{i=0}^{\infty} e^{-\Gamma_{i} t} r_{i}(x) r_{i}(y)
$$

and also because $\Gamma_{0}=0$ with $r_{0}(x)=\frac{1}{\sqrt{V_{1}(M)}}$,

$$
G(x, y, t)=\sum_{i=1}^{\infty} e^{-\Gamma_{i} t} r_{i}(x) r_{i}(y)
$$

It is easy to check that $G$ satisfies the semi-group property

$$
G(x, y, t+s)=\int_{M} G(x, z, t) G(z, y, s) u_{1}^{\frac{-4}{m-2}} d v_{1}(z)
$$

for $x, y \in M$, and $t, s \in[0, \infty)$. Moreover

$$
\int_{M} G(x, y, t) u_{1}^{-\frac{4}{m-2}}(y) d v_{1}(y)=0
$$

because $\int_{M} r_{i}(y) u_{1}^{\frac{-4}{m-2}} d v_{1}(y)=\int_{M} u_{i}(y) u_{1}(y) d v(y)=0$, for $i \geq 1$. Also

$$
\int_{M}|G(x, y, t)| u_{1}^{\frac{-4}{m-2}} d v_{1}(y) \leq \int_{M}|W(x, y, t)| u_{1}^{\frac{-4}{m-2}} d v_{1}(y)+1=2
$$

because $W(x, y, t)>0$ and $\int_{M} W(x, y, t) u_{1}^{\frac{-4}{m-2}} d v_{1}=1$.

To prove the theorem, we first estimate $G(x, x, t)$ from above. By $(28)$ and (30)

$$
\begin{aligned}
G^{\prime}(x, x, 2 t) & =2 \int_{M} G^{\prime}(x, y, t) G(x, y, t) u_{1}^{\frac{-4}{m-2}} d v_{1} \\
& =2 \int_{M} \Delta_{1} G(x, y, t) G(x, y, t) d v_{1} \\
& =-2 \int_{M}\left|\nabla_{1} G(x, y, t)\right|^{2} d v_{1} \\
& \leq-2^{1-\frac{4}{m}} \alpha_{13}\left(\int_{M} G^{2}(x, y, t) d v_{1}\right)^{\frac{m+2}{m}} .
\end{aligned}
$$


However the semi-group property (28) of $G$ implies that

$$
-G^{\prime}(x, x, t)(G(x, x, t))^{-\frac{m+2}{m}} \geq 2^{-\frac{4}{m}} \alpha_{13} .
$$

Integrating both sides of (32) with respect to $t$, and using the fact that

$$
G(x, x, t)=(W(x, x, t)-1) \rightarrow \infty
$$

as $t \rightarrow 0$, we have

$$
\frac{m}{2} G^{-\frac{2}{m}}(x, x, t) \geq 2^{-\frac{4}{m}} \alpha_{13} t
$$

Hence,

$$
G(x, x, t) \leq 4\left(\frac{m}{2 \alpha_{13}}\right)^{\frac{m}{2}} t^{-\frac{m}{2}}
$$

Integrating both sides with respect to $x$ and using the expansion (27), we have

$$
\begin{aligned}
\sum_{i=1}^{\infty} e^{-\Gamma_{i} t} & \leq 4\left(\frac{m}{2 \alpha_{13}}\right)^{\frac{m}{2}} t^{-\frac{m}{2}} V_{1}(M) \\
& \leq 4 C_{h}^{-\frac{2 m}{m-2}} V(M)^{-\frac{2}{m-2}}\left(\frac{m}{2 \alpha_{13}}\right)^{\frac{m}{2}} t^{-\frac{m}{2}} .
\end{aligned}
$$

Let $t=\frac{1}{\Gamma_{k}}$, and observe that $\frac{\Gamma_{i}}{\Gamma_{k}} \leq 1$ for $i \leq k,(35)$ becomes

$$
\begin{aligned}
4 C_{h}^{-\frac{2 m}{m-2}} V(M)^{-\frac{2}{m-2}}\left(\frac{m}{2 \alpha_{13}}\right)^{\frac{m}{2}}\left(\frac{1}{\Gamma_{k}}\right)^{-\frac{m}{2}} & \geq \sum_{i=1}^{\infty} e^{-\frac{\Gamma_{i}}{\Gamma_{k}}} \\
& \geq \sum_{i=1}^{k} e^{-\frac{\Gamma_{i}}{\Gamma_{k}}} \\
& \geq k e^{-1} .
\end{aligned}
$$

This proves the theorem.

\section{References}

[B] P. Buser, A note on the isoperimetric constant, Ann. Sci. École Norm. Sup., 15(4) (1982), 213-230.

[C] J. Cheeger, A lower bound for the smallest eigenvalue of the Laplacian, Problem in Analysis, Princeton Univ. Press, (1970), 195-199. 
[Cn1] R. Chen, Neumann eigenvalue estimate on a compact Riemannian manifold, Proc. AMS, 108 (1990), 961-970.

[Cn2] On global Schrödinger kernel estimate and eigenvalue problem, to appear, Math. Z.

[CnL] R. Chen and P. Li, On Poincaré type inequalities, to appear, Trans. AMS.

[CL] S.Y. Cheng and P. Li, Heat kernel estimates and lower bounds of eigenvalues, Comment. Math. Helv., 56 (1981), 327-338.

[L1] P. Li, A lower bound for the first eigenvalue of the Laplacian on a compact Riemannian manifold, Indiana U. Math. J., 28 (1979), 1013-1019.

[L2] Poincaré inequalities on Riemannian manifolds, Seminar on Differential Geometry (104), ed. S.T. Yau, Princeton Univ. Press, (1982), 73-84.

[L3] On the Sobolev constant and the p-spectrum of a compact Riemannian manifold, Ann. Sci. École Norm. Sup., 13 (1980), 451-469.

[L4] Lecture notes on geometric analysis, Lec. notes Ser. 6, Res. Inst. Math., Seoul Nat'l Univ. Press, 1993.

[LY1] P. Li and S.T. Yau, Estimates of eigenvalues of a compact Riemannian manifold, AMS Proc. Symp. Pure Math., 36 (1980), 205-239.

[LY2] - On the parabolic heat kernel of the Schrödinger operator, Acta Math., 156 (1986), 153-201.

[M] D. Meyer, Minoration de la première valeur propre non nulle de problème de Neumann sur les variétés Riemanniennes à bord, Ann. Inst. Fourier, Grenoble, 36 (1986), 113-125.

[PW] L.E. Payne and H. Weinberger, The optimal Poincaré inequality for convex domains, Arch. Rational Mech. Anal., 5 (1960), 282-292.

[SWYY] I.M. Singer, B. Wong, S.S.T. Yau and S.T. Yau, An estimate of the gap of the first two eigenvalues of the Schrödinger operator, Ann. Scuo. Norm. Pisa, Cl. Sci., IV(12) (1985), 319-333.

[W] J. Wang, Global heat kernel estimates, preprint, 1994.

[ZY] J.Q. Zhong and H.C. Yang, On the estimate of first eigenvalue of a compact Riemannian manifold, Sci. Sinica Ser., 27(A) (1984), 1265-1273.

Received August 22, 1995 and revised October 14, 1995.

National Cheng-Kung University

TAINAN 70101, TAIWAN

E-mail address: rchen@mail.ncku.edu.tw 